\title{
Increased Dopamine Level Enhances Male-Male Courtship in Drosophila
}

\author{
Tong Liu, ${ }^{1,2}$ Laurence Dartevelle, ${ }^{4}$ Chunyan Yuan, ${ }^{1}$ Hongping Wei, ${ }^{1,2}$ Ying Wang, ${ }^{1}$ Jean-François Ferveur, ${ }^{4}$ and \\ Aike Guo ${ }^{1,3}$ \\ ${ }^{1}$ Institute of Neuroscience, State Key Laboratory of Neuroscience, Shanghai Institutes for Biological Sciences, Chinese Academy of Sciences, Shanghai \\ 200031, China, ${ }^{2}$ Graduate School of Chinese Academy of Sciences, Beijing 100039, China, ${ }^{3}$ State Key Laboratory of Brain and Cognitive Science, Institute of \\ Biophysics, Chinese Academy of Sciences, Beijing 100101, China, and ${ }^{4}$ Centre National de la Recherche Scientifique, Unité Mixte de Recherche 5548, \\ Universite de Bourgogne, Dijon 21000, France
}

Sexual behavior between males is observed in many species, but the biological factors involved are poorly known. In mammals, manipulation of dopamine has revealed the role of this neuromodulator on male sexual behavior. We used genetic and pharmacological approaches to manipulate the dopamine level in dopaminergic cells in Drosophila and investigated the consequence of this manipulation on male-male courtship behavior. Males with increased dopamine level showed enhanced propensity to court other males but did not change their courtship toward virgin females, general olfactory response, general gustatory response, or locomotor activity. Our results indicate that the high intensity of male-male interaction shown by these manipulated males was related to their altered sensory perception of other males.

Key words: Drosophila; courtship; male-male courtship behavior; neurotransmitter; tyrosine hydroxylase; dopamine

\section{Introduction}

In nature, animals use multiple sensory cues to recognize conspecifics and to choose a potentially suitable mate for reproduction (Bradbury and Vehrencamp, 1998; Wyatt, 2003). In Drosophila melanogaster, courtship behavior, which precedes mating, mostly depends on visual, acoustic, and chemosensory signals exchanged by the two partner flies that alternatively and reciprocally act as the courter and as the courtee (Hall, 1994; Yamamoto et al., 1997; Lasbleiz et al., 2006). Some of these sensory signals can stimulate male or female courtship, whereas others can inhibit sexual behavior (Greenspan and Ferveur, 2000). Although wild-type male flies rarely show male-male courtship, the frequency and intensity of this behavior can be strongly increased by genetic manipulation (Greenspan and Ferveur, 2000). For example, substantial male-male courtship has been found in flies with mutation of fruitless ( fru) (Hall, 1994; Yamamoto et al., 1996), dissatisfaction (Finley et al., 1997), prospero (Balakireva et al.,

Received Sept. 3, 2007; revised March 9, 2008; accepted April 14, 2008.

This research was supported by National Science Foundation of China Grants 30270341, 30630028, and 30621004 (A.G), the Multidisciplinary Research Program (Brain and Mind) of the Chinese Academy of Sciences, Major State Basic Research Program Grant G20000 77800, 2006CB806600, and 2006CB911003 (A.G), the Precedent Project of Important Intersectional Disciplines in the Knowledge Innovation Engineering Department of the Chinese Academy of Sciences Grants KJCX1-09-03 and KSCX2-YW-R-28 (A.G), and by Centre National de la Recherche Scientifique and the Burgundy Regional Council (L.D. and J.-F.F.). We thank M.-M. Poo, R. Greenspan, and three anonymous reviewers for helpful comments, S. Carroll for the UAS-TH line, and J. Hirsh for the TH-GAL4 line.

Correspondence should be addressed to either of the following: Aike Guo, Institute of Neuroscience, State Key Laboratory of Neuroscience, Shanghai Institutes for Biological Sciences, Chinese Academy of Sciences, Shanghai 200031, China, E-mail: akguo@ion.ac.cn; or Jean-François Ferveur, Centre National de la Recherche Scientifique, Unité Mixte de Recherche 5548, Universite de Bourgogne, Dijon 21000, France, E-mail: jean-francois.ferveur@u-bourgogne.fr.

DOI:10.1523/JNEUROSCI.5290-07.2008

Copyright $\odot 2008$ Society for Neuroscience $\quad 0270-6474 / 08 / 285539-08 \$ 15.00 / 0$
1998), or quick-to-court (Gaines et al., 2000) genes. The ectopic expression of a female-dominant form of the transformer gene $\left(\operatorname{tra}^{F}\right)$ (Ferveur et al., 1995; O'Dell et al., 1995) and the presence of the mini-white transgene ( $m w$ ) (Zhang and Odenwald, 1995; Hing and Carlson, 1996) are also associated with male-male courtship. Several brain regions involved in male-male courtship behavior have been identified by targeted expression of $\operatorname{tra}^{F}$ and fru in male brains under the control of specific galactosidase- 4 (GAL4) lines (Ferveur et al., 1995; Billeter et al., 2006). For example, when $\mathrm{tra}^{F}$ was expressed in either antennal lobes or in mushroom bodies, feminized male flies showed high male-male courtship behavior (Ferveur et al., 1995; O’Dell et al., 1995). The genetic alteration of either a subset of peripheral taste neurons or glial cells located in the olfactory centers of the brain can also affect male-male courtship without altering male-female courtship (Lacaille et al., 2007; Grosjean et al., 2008).

In mammals, male sexual behavior is regulated by several neuromodulators, including dopamine (DA) and serotonin (5-HT). Pharmacological manipulation of DA or 5-HT systems in mammalian can alter their sexual behavior. These two substances seem to exert reciprocal effects, with DA facilitating and 5-HT inhibiting male sexual behavior (Melis and Argiolas, 1995; Hull et al., 2004). Although the possible effect of 5-HT on Drosophila male sexual orientation was discussed (Zhang and Odenwald, 1995) and DA was shown to modulate male arousal and visual perception during heterosexual courtship (Andretic et al., 2005; Kume et al., 2005), locomotor activity (Pendleton et al., 2002), female sexual receptivity (Neckameyer, 1998a), male courtship conditioning (Neckameyer, 1998b), and ethanol-induced courtship disinhibition (Lee et al., 2008), the effect of DA on male-male courtship behavior remains poorly known. 
Here, we used genetic and pharmacological tools to modulate DA level in DA cells. We evaluate the effect of these manipulations on both the DA level in male brain and the intensity of Drosophila male courtship behavior in relation with sensory perception. Our results showed that increased DA level was correlated with a more intense male-male courtship toward other mature males.

\section{Materials and Methods}

Fly stocks. The wild-type strain used was Canton S. The GAL4 driver strains used were tyrosine hydroxylase (TH)-GAL4 (Friggi-Grelin et al., 2003a), 201Y-GAL4, choline acetyltransferase (Cha)-GAL4 (Salvaterra and Kitamoto, 2001), and the pan-neuronal expression line elav (embryonic lethal, abnormal vision, Drosophila)-GAL4 (Robinow and White, 1988). The upstream activating sequence (UAS) reporter strains used were UAS-TH (True et al., 1999) and UAS-enhanced green fluorescent protein $(E G F P)$. The mutant strain used was ebony $\left(e^{l}\right)$ (Ramadan et al., 1993). The Oregon- $R$ strain was used as the control of ebony (Ramadan et al., 1993).

Fly culture and surgery. Flies within $4 \mathrm{~h}$ after eclosion were collected under light $\mathrm{CO}_{2}$ anesthesia and then transferred into unyeasted standard medium (Bloomington Stock Center) eventually supplemented with different drugs. Flies were reared either at $18^{\circ} \mathrm{C}$ or $25^{\circ} \mathrm{C}$, with $60 \%$ relative humidity, and a $12 \mathrm{~h}$ light/dark photoperiod. Male and female flies used in behavioral assays were aged for $5-8$ and $2-4 \mathrm{~d}$ after eclosion, respectively. For some experiments, the males' antennae and maxillary palps were ablated (NAM) when the flies were $1 \mathrm{~d}$ old, and the experiments were conducted $7 \mathrm{~d}$ later. We used the following drug concentrations: (1) to decrease the DA level in the brain of TH-GAL4/UAS-TH males, 0.1 mu reserpine (Res; Sigma), $0.5 \mathrm{mg} / \mathrm{ml} \alpha$-methyl-DL-tyrosine methyl ester hydrochloride (AMPT; Sigma), 10 mM SKF 83566 (8-bromo-2,3,4,5tetrahydro-3-methyl-5-phenyl-1H-3-benzazapin-7-ol hydrobromide; Tocris Bioscience), and 10 and $30 \mathrm{~mm}$ raclopride (Tocris Bioscience); (2) to increase DA level in wild-type males, $10 \mathrm{mg} / \mathrm{ml} \mathrm{L-3,4-}$ dihydroxyphenylalanine (L-DOPA; Sigma) and 0.1\% L-ascorbic acid (Sigma). Reserpine was dissolved in DMSO (Sigma; $0.5 \mathrm{ml}$ DMSO/100 $\mathrm{ml}$ media) first and then added into the medium. Other drugs were directly added into the medium when the medium was cooling down to $60-70^{\circ} \mathrm{C}$. L-Ascorbic acid was used as antioxidant in L-DOPA containing medium. For all drug treatments, flies were collected $<4 \mathrm{~h}$ after eclosion and raised on drug-containing food for 6 or $7 \mathrm{~d}$ until the behavior assays were performed.

Behavior. Paired courtship assay was performed in a courtship chamber $(12 \times 18 \mathrm{~mm})$ with a pair of flies. The courtship index $(\mathrm{CI})$ is the percentage of time in a 5 min observation period that the tester male spent courting the target fly (this includes tapping, following, wing vibration, and attempted copulation) (Villella et al., 1997). When both flies had the same appearance, they were distinguished with a color dot painted on the thorax of the target flies, few minutes before the experiment. Tester males were reared individually, whereas target flies were reared in groups of $40-50$.

Competitive courtship or courtship preference assay was performed with a single tester male simultaneously presented to two decapitated target flies, a wild-type male and a wild-type female, in a courtship chamber. The CIs toward the female and the male were simultaneously measured, and then these CIs were compared. Tester males were raised individually (Villella et al., 1997).

Chaining assay involved 10 flies introduced in a $95 \times 23 \mathrm{~mm}$ tube with fresh food (Villella et al., 1997). A chain was defined as a group of at least three males exhibiting courtship behavior to each other (Finley et al., 1997). Chaining index (ChI) is the percentage of time that groups of males spent on courting during a 10 min observation period. Males were reared in groups of 60-70.

Locomotor activity was measured by placing single flies in a courtship chamber, containing a filter paper labeled with a single bisecting line. The total number of lines crossed within a 5 min observation period indicates the locomotor activity (Villella et al., 1997).

Olfactory sensitivity was measured by avoidance of different concen- trations of 4-methylcyclohexanol (MCH; Fluka) and 3-octanol (OCT; Aldrich) using the method of Anholt et al. (1996).

Proboscis extension response was used to test the gustatory sensitivity. Single 5-d-old females and males were starved for $24 \mathrm{~h}$, anesthetized with $\mathrm{CO}_{2}$, then fixed on slides with tape and modeling clay, and left for $2 \mathrm{~h}$ in a humid box to recover. Proboscis extension was elicited by touching the tarsi of the forelegs with a drop of sucrose solution. Each fly was tested five times by using the same sucrose concentration. To avoid habituation, we waited at least $30 \mathrm{~s}$ between tests (Heimbeck et al., 2001).

Determination of DA content in brains. The DA level was measured as by Ye et al. (2005). Briefly, the heads were removed and dissected freshly in PBS. Then, the brains were homogenized in $0.1 \mathrm{~m}$ perchloric acid immediately, and the homogenate was centrifuged at $12,000 \times g$ at $4^{\circ} \mathrm{C}$ for $30 \mathrm{~min}$. The supernatant fluid was used to measure DA content with an ${ }^{125}$ I-radioimmunoassay dopamine kit (LDN).

\section{Results \\ Overexpression of tyrosine hydroxylase in Drosophila DA cells enhanced male-male courtship}

Initially, we found that mature male flies overexpressing $\mathrm{TH}$ (the rate-limiting enzyme of DA synthesis) in DA cells (TH-GAL4/ UAS-TH line) showed increased frequency of male-male interaction. These males carried two transgenes: (1) TH-GAL4 known to drive GAL4 expression in most DA cells during all the developmental stages (Friggi-Grelin et al., 2003a,b), and (2) UAS-TH, which allows the overexpression of $\mathrm{TH}$ in the tissues in which GAL4 is expressed (Brand and Perrimon, 1993; True et al., 1999). When TH-GAL4/UAS-TH males were tested in groups, they frequently formed courtship chains or rings (Fig. 1A,B) (supplemental Movie S1, available at www.jneurosci.org as supplemental material). The duration of this behavior was quantified with the ChI (Fig. 1B) (Villella et al., 1997). Moreover, when these males were paired, they showed an intense male-male courtship, and its duration was quantified as the CI (Fig. 2A) (supplemental Movie S2, available at www.jneurosci.org as supplemental material) (Villella et al., 1997). Only the combination of both transgenes induced this behavioral effect because males carrying only one copy of either transgene did not show increased male-male interaction $(T H-G A L 4 /+$ and +/UAS-TH) (Figs. $1 B, 2 A)$. Moreover, overexpression of TH in DA cells did not change male overall courtship toward females (Fig. 2A). This indicates that the genetic manipulation did not change the sexual orientation of male flies (e.g., heterosexual vs homosexual) but rather increased the male propensity to court other males without altering their courtship of virgin females.

Given that TH-GAL4/UAS-TH tester males directed high CIs toward male and female target flies tested separately, we measured their ability to discriminate flies of both sexes by using a competitive courtship assay (or preference courtship assay) in which we measured the CI that single tester males directed toward each of the two decapitated control target flies: one female and one male, simultaneously presented to the tester male, under white light. The clear preference that TH-GAL4/UAS-TH males showed to females (Fig. 2C) $(p<0.001$, Mann-Whitney rank sum test) indicates that they have a reduced but still strong ability to discriminate the sexes. Although the total CI (the sum of CIs toward males and females) was not different between the two male genotypes, TH-GAL4/UAS-TH males showed higher male-male courtship, and decreased male-female courtship, than wild-type males (Fig. $2 C)(p<0.001$ and $p=0.045$, respectively).

The targeted expression of UAS-TH driven by other GAL4 lines induced variable effects: when $\mathrm{TH}$ was overexpressed in a pan-neuronal pattern (elav-GAL4/UAS-TH line), a high ChI was 


\section{A}

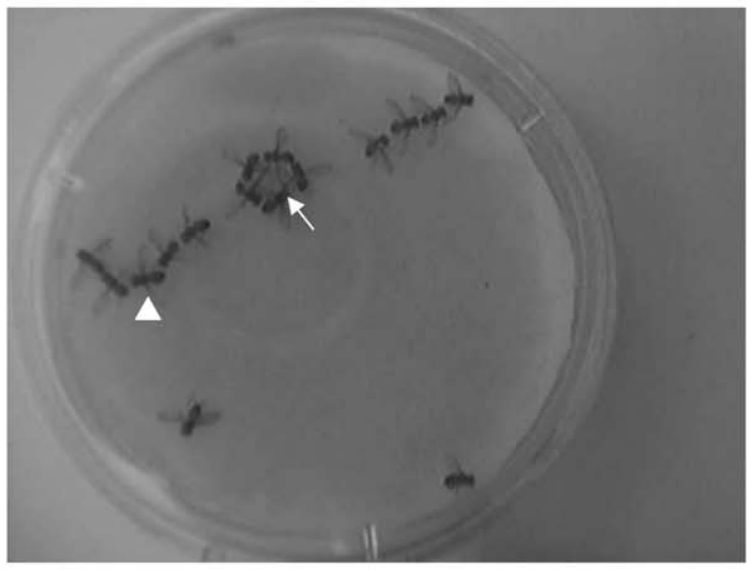

B

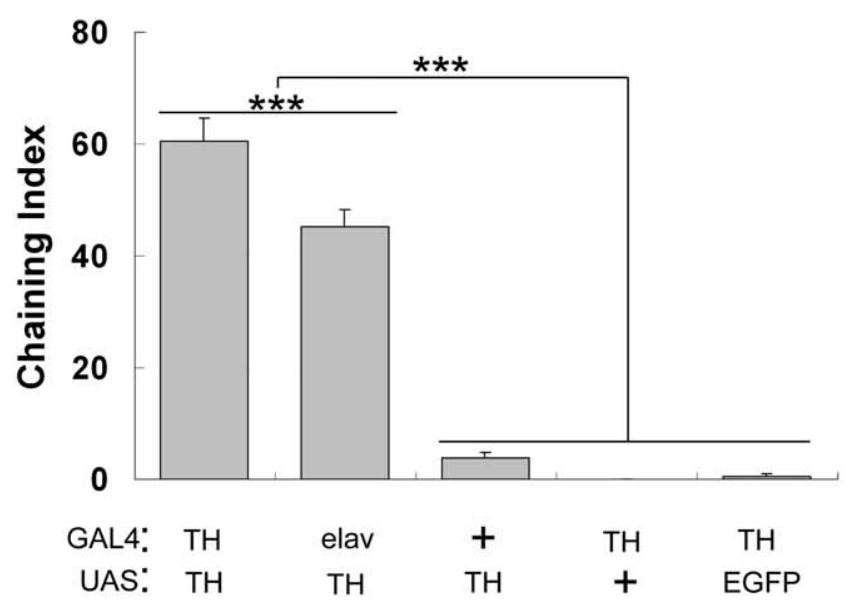

C

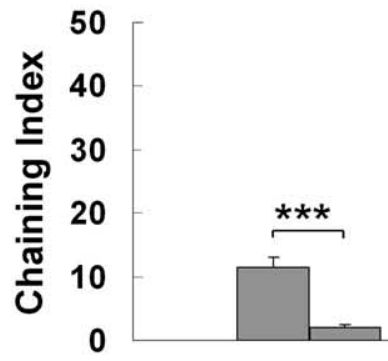

Genotype:

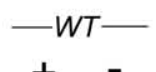

DOPA:

Figure 1. Genetic targeting of TH and increased male-male interactions. A, TH-GAL4/ UAS-TH males frequently formed chains (arrowhead) and rings (arrow), revealing strong male-male interactions. $\boldsymbol{B}$, Specific overexpression of TH in DA cells induced strong male $\mathrm{Chl}$. $\mathrm{Chl}$ is shown for various male genotypes involving a GAL4 driver and/or a UAS reporter gene (GAL4/UAS). From left to right, TH was overexpressed in DA cells (TH/TH), in a pan-neuronal pattern (elav/TH); only the UAS-TH transgene was present (+/TH); only the TH-GAL4 transgene was present (TH/+); EGFP was expressed in DA cells (TH/EGFP). $n=27-51$. C, DA level was increased in either wild-type males (WT) by feeding with L-DOPA or in the ebony mutants $\left(e^{T}\right)$. Oregon- $R($ Or- $R$ ) is the control for ebony. $n=29-56$, except for Oregon- $R(n=17)$. Each histogram represents the mean $\mathrm{Chl}$ with its SEM. ${ }^{* * *} p<0.001$ (for two-group comparison, Mann-Whitney rank sum test was used; for more than two-group comparison, ANOVA on rank test was used).

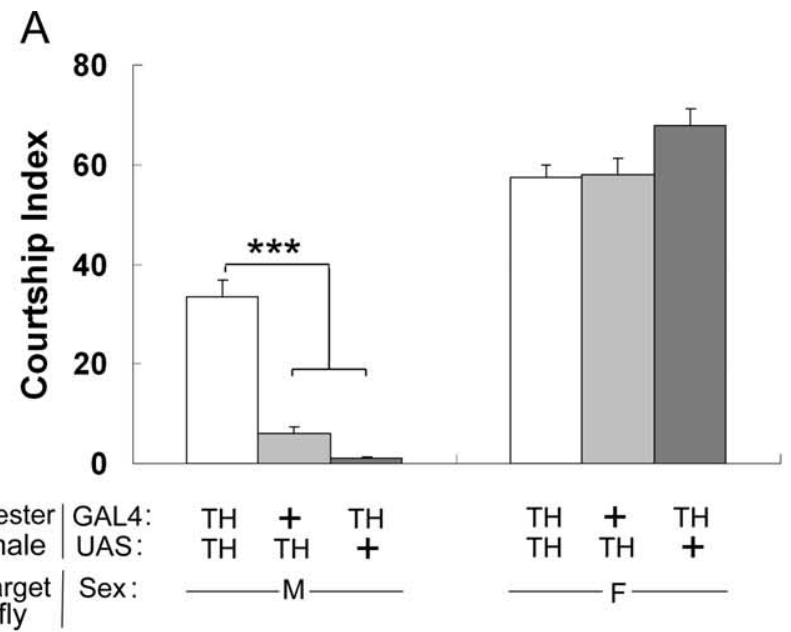

B
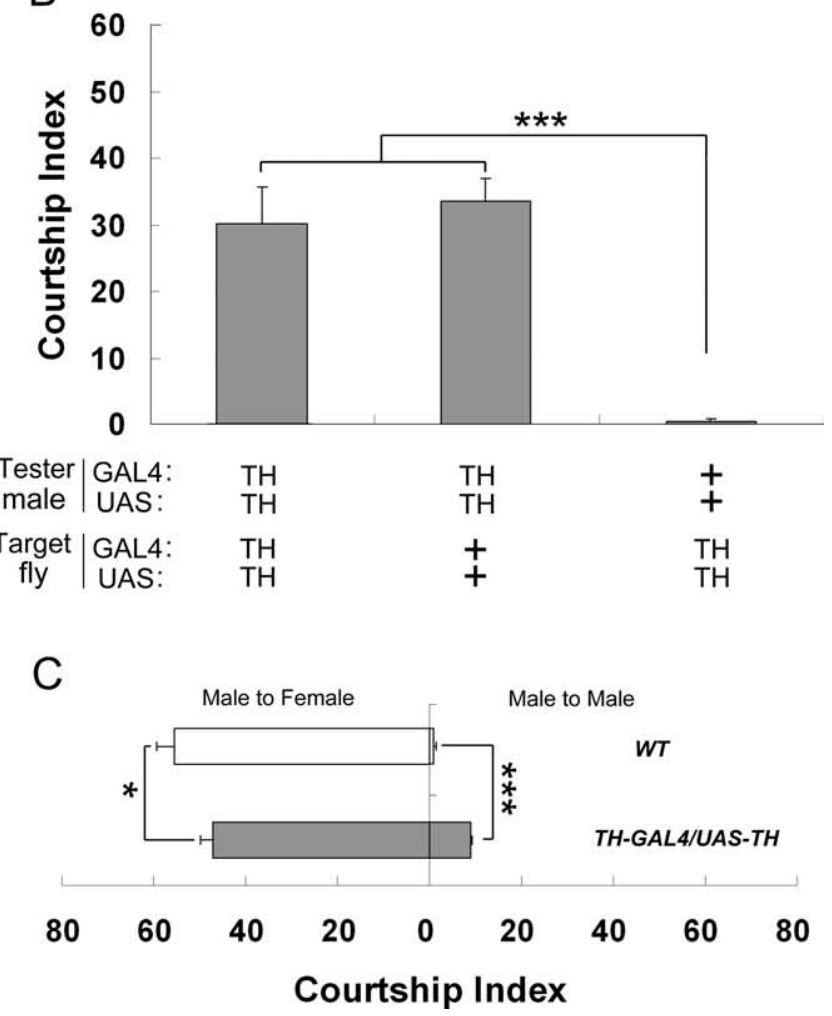

Figure 2. Increased male-male courtship of TH-GAL4/NAS-TH males. $\boldsymbol{A}, T H-G A L 4 /$ UAS-TH tester males courted wild-type target males (M) much more actively than control transgenic testers ( $+/$ UAS-TH and $T H-G A L 4 /+$ ). Courtship of these testers to wild-type target females was also measured (F). $n=34-39 . B, T H-G A L 4 / N A S-T H$ tester males showed enhanced propensity to court wild-type target males, but wild-type tester males did not court TH-GAL4/NAS-TH target males. $n=34$ except for homotypic pairs $(n=16)$. C, Courtship of TH-GAL4/UAS-TH males in competitive courtship assay. TH-GAL4/NAS-TH males showed significantly higher male-male $\mathrm{Cl}$ and lower male-female $\mathrm{Cl}$ compared with that shown by wildtype males. $n=69$ for $T H-G A L 4$ NAS-TH males and $n=36$ for wild-type males. Each histogram represents the mean $\mathrm{Cl}$ with its SEM. ${ }^{*} p<0.05$ and ${ }^{* * *} p<0.001$ (for two-group comparison, Mann-Whitney rank sum test was used; for more than two-group comparison, ANOVA on rank test was used).

induced (Fig. $1 B$ ). However, ChI did not increase when $\mathrm{TH}$ was overexpressed (1) in the mushroom bodies, a central brain structure crucial for many complex behaviors, including male courtship behavior (201Y/UAS-TH line; ChI of $1.77 \pm 0.6, n=52$ ) (Ferveur et al., 1995; Neckameyer, 1998b; Heisenberg, 2003), or 
(2) in cholinergic neurons (Cha-GAL4/UAS-TH line; ChI of $2.88 \pm 1.7, n=49)$, which are involved in several aspects of male sexual behavior (Kitamoto, 2002; Acebes et al., 2004). Moreover, overexpression of EGFP in DA cells ( TH-GAL4/UAS-EGFP line) induced no behavioral effect (Fig. $1 B$ ). These experiments indicate that the high level of male-male interaction was related to the overexpression of TH in DA cells of the nervous system. This allows us to exclude the possibility that the high level of malemale courtship observed in our experiment was caused by either TH overexpression in any tissue or an unspecific effect in DA cells like that resulting from the misexpression of the mini-white gene (Zhang and Odenwald, 1995). This also excluded the possibility that male-male courtship results from the epigenetic interaction between the GAL4 and UAS transgenes because their combination did not always result in a high male-male courtship behavior.

Given that the male-male interaction between two $\mathrm{TH}-$ GAL4/UAS-TH males could be caused by a reciprocal defect in the courtship directed and/or induced by each male to his partner, we tested both these possibilities. Practically, we measured the amount of courtship behavior (CI) that individual $\mathrm{TH}-$ GAL4/UAS-TH males either directed or induced to single wildtype males (Fig. 2 B). Our data showed that, although TH-GAL4/ UAS-TH males directed an intense CI to control males, the reciprocal interaction (the $\mathrm{CI}$ that transgenic males induced to wild-type males) was very low and similar to the CI induced by control males. This indicates that TH-manipulated males have an altered propensity to court other males but not to be courted by wild-type males.

Because male-male interaction shown by TH-GAL4/ UAS-TH flies could be attributable to a general olfactory/gustatory defect, we performed experiments to measure whether $\mathrm{TH}-$ GAL4/UAS-TH males has a general olfactory or gustatory defect. To test olfactory sensitivity, we used different concentration of $\mathrm{MCH}$ and OCT to compare the odor sensitivity between $\mathrm{TH}-$ GAL4/UAS-TH and control males (Anholt et al., 1996). To test gustatory sensitivity, we used the proboscis extension response for different concentration of sucrose (Heimbeck et al., 2001). The fact that no significant difference of odor and taste sensitivity was observed between the two male genotypes (Fig. 3) indicates that the increased intensity of male-male interaction induced by the overexpression of $\mathrm{TH}$ in DA cells was not attributable to a general olfactory/gustatory defect.

Together, these results indicate that the genetic overexpression of TH in DA cells strongly changed male propensity to court other males but did not affect their attractiveness to other males nor their general olfactory/gustatory and locomotor activities.

\section{Correlation between the DA level and the intensity of male- male courtship behavior}

Because TH is the rate-limiting enzyme for DA synthesis, we checked by radioimmunoassay the level of DA in the brain of transgenic males (Fig. 4). DA level was significantly higher in TH-GAL4/UAS-TH males than that in control transgenic males ( $T H-$ GAL4/+ and +/UAS-TH). To explore the relationship between DA content in the brain and male-male courtship behavior, we fed TH-GAL4/UAS-TH males during their adulthood with two drugs that can reduce DA content: (1) Res, an inhibitor of vesicular monoamine transporter, and (2) AMPT, a potent inhibitor of TH (Pendleton et al., 2005).

First, we found significantly less DA in the brain of TH-GAL4/ UAS-TH males fed with either Res- or AMPT-containing medium than in that of sibling males fed on standard medium (Fig.
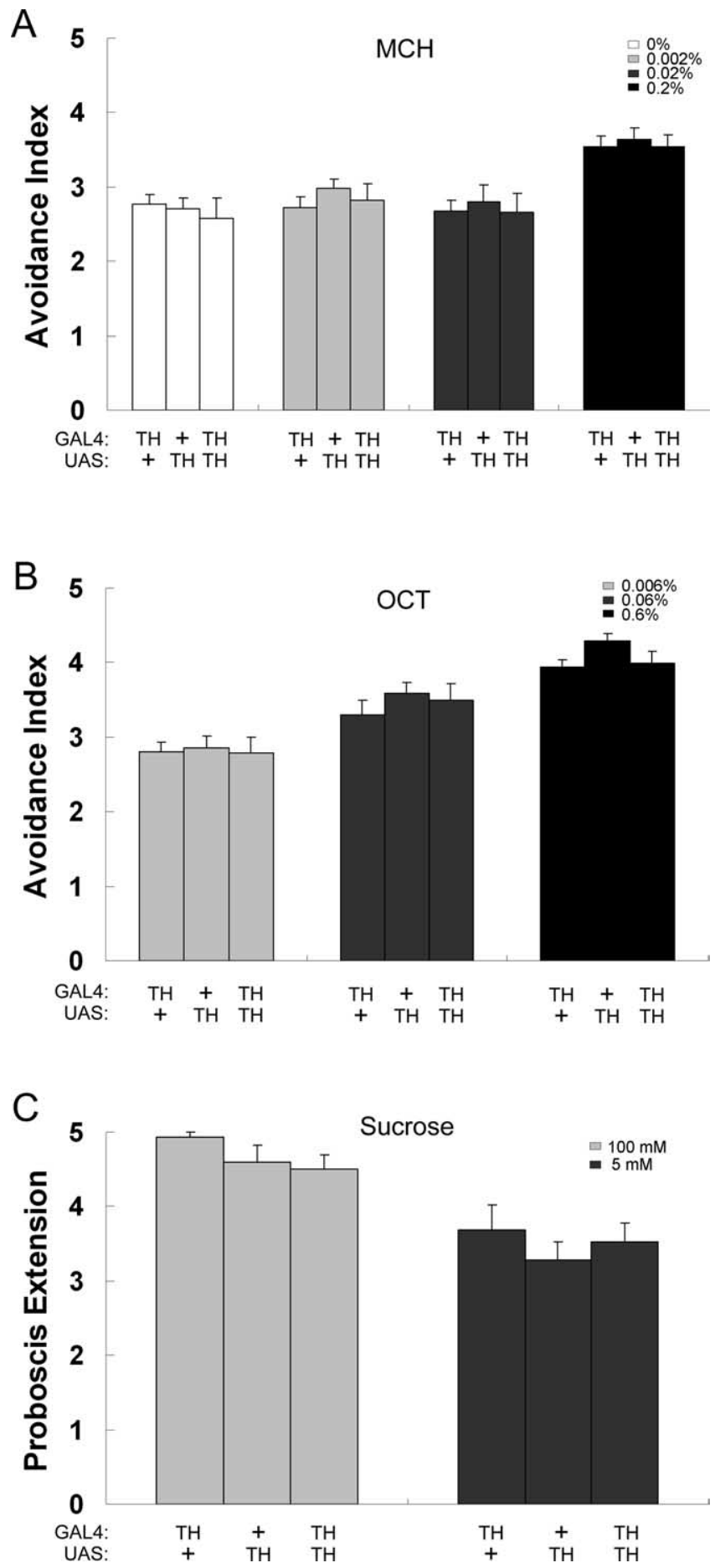

Figure 3. Odor/taste sensitivity of TH-GAL4/NAS-TH and control males. A, Different concentrations of $\mathrm{MCH}$ were used to measure the ability of olfactory detection of $T H-G A L 4 / N A S-T H$ males and of control transgenic males. $n=11-16 . B$, Different concentrations of OCT were used to compare the olfactory response of TH-GAL4/NAS-TH males and control males. $n=$ 15-20. C, Different concentrations of sucrose were used to test the ability of gustatory detection of TH-GAL4/NAS-TH males and of control transgenic males. $n=15-30$. Each histogram represents the mean behavioral activity with its SEM. Statistics was performed with one-way ANOVA test, and no difference between genotypes was detected.

4). Second, both the ChI (Fig. 5A) and the male-male CI (Fig. 5B) of Res- or AMPT-fed TH-GAL4/UAS-TH males was also dramatically reduced compared with that of untreated sibling males. Although both Res and AMPT treatments changed male-male 


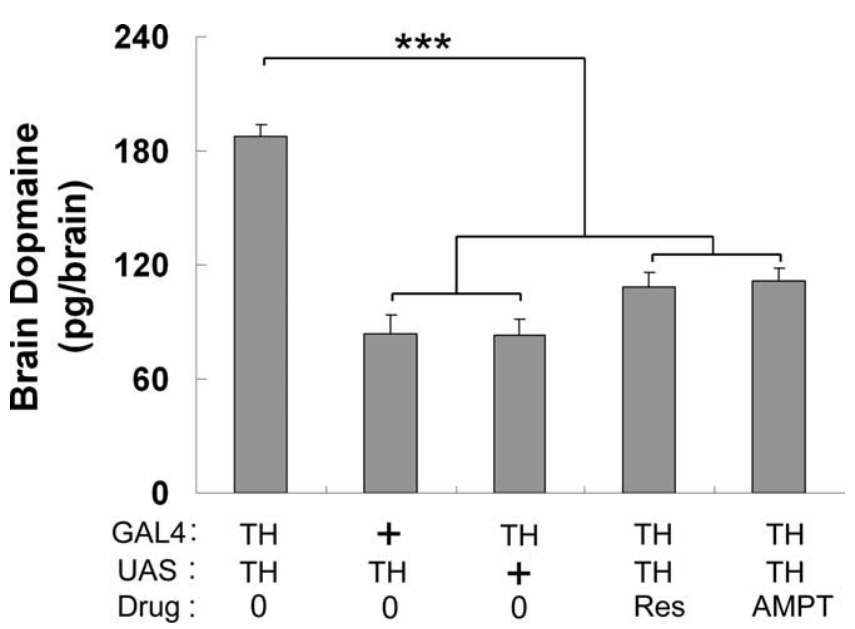

Figure 4. DA level in the brain of various males. Data showed the DA level (picograms per brain) in complete brains. DA level in TH-GAL4/NAS-TH males fed on regular food (0) or food added with reserpine (Res) or with AMPT. DA level is also shown for single transgenic (UAS$T H /+$ and $T H-G A L 4 /+$ ) males. $n=7-9$. Each histogram represents the mean DA level with its SEM. ${ }^{* * *} p<0.001$ (one-way ANOVA test was used).

interaction, they affected neither male overall courtship toward wild-type females (Fig. 5B) nor male spontaneous locomotor activity (Fig. 5C).

To further confirm the pharmacological effect on male-male courtship behavior shown by TH-GAL4/UAS-TH males, we used the tubulin promoter $t u b-G A L 80^{\text {ts }}$ to decrease DA level of TH-GAL4/UAS-TH male ( $t u b-G A L 80^{\text {ts }} /+$; TH-GAL4/UAS$\mathrm{TH}$ ). When they were raised at $18^{\circ} \mathrm{C}$ (the permissive temperature at which GAL80 ${ }^{\text {ts }}$ represses GAL4) (McGuire et al., 2003), tubGAL80 ${ }^{\text {ts }} /+$; TH-GAL4/UAS-TH males showed a significantly decreased values compared with that of TH-GAL4/UAS-TH males for both (1) the DA level in their brain (respectively, $91.08 \pm 7.2 \mathrm{pg}, n=8$ and $119.80 \pm 7.9 \mathrm{pg}, n=7 ; p=0.018$, Student's $t$ test) and (2) their ChI values (respectively, $12.63 \pm$ $1.7, n=67$ and $32.98 \pm 4.0, n=41 ; p<0.001$, Mann-Whitney rank sum test).

The experiments below also suggest that DA variation affects the intensity of male-male courtship behavior. Wild-type males fed with L-DOPA (the precursor for DA synthesis) (Pendleton et al., 2005) after adult eclosion showed significantly increased ChI compared with sibling males continuously raised in standard medium (Fig. 1C). Moreover, the ChI of ebony $\left(e^{l}\right)$ mutant males in which DA accumulates since early development (Ramadan et al., 1993) was much higher than that of wild-type flies sharing the same genetic background (without the $e^{l}$ mutation; Oregon- $R$ ) (Fig. 1C).

The ChI of the flies with genetic manipulation during the whole development (TH targeting, $e^{l}$ mutation) was stronger than that of flies fed with L-DOPA during adulthood. This suggests that DA manipulation exerts additive effects on behavior during both preimaginal and adult development, because the wild-type males fed with L-DOPA even showed higher brain DA content compared with that of TH-GAL4/UAS-TH males (data not shown). These results reinforce the positive relationship between the increased DA level in the brain and the male propensity to court other males.

To investigate more precisely the role of DA signaling on male-male courtship behavior, we tested the effects of two substances known for their antagonistic effects on DA receptors: SKF 83566, the antagonist of $\mathrm{D}_{1}$-like receptors (Yellman et al., 1997),
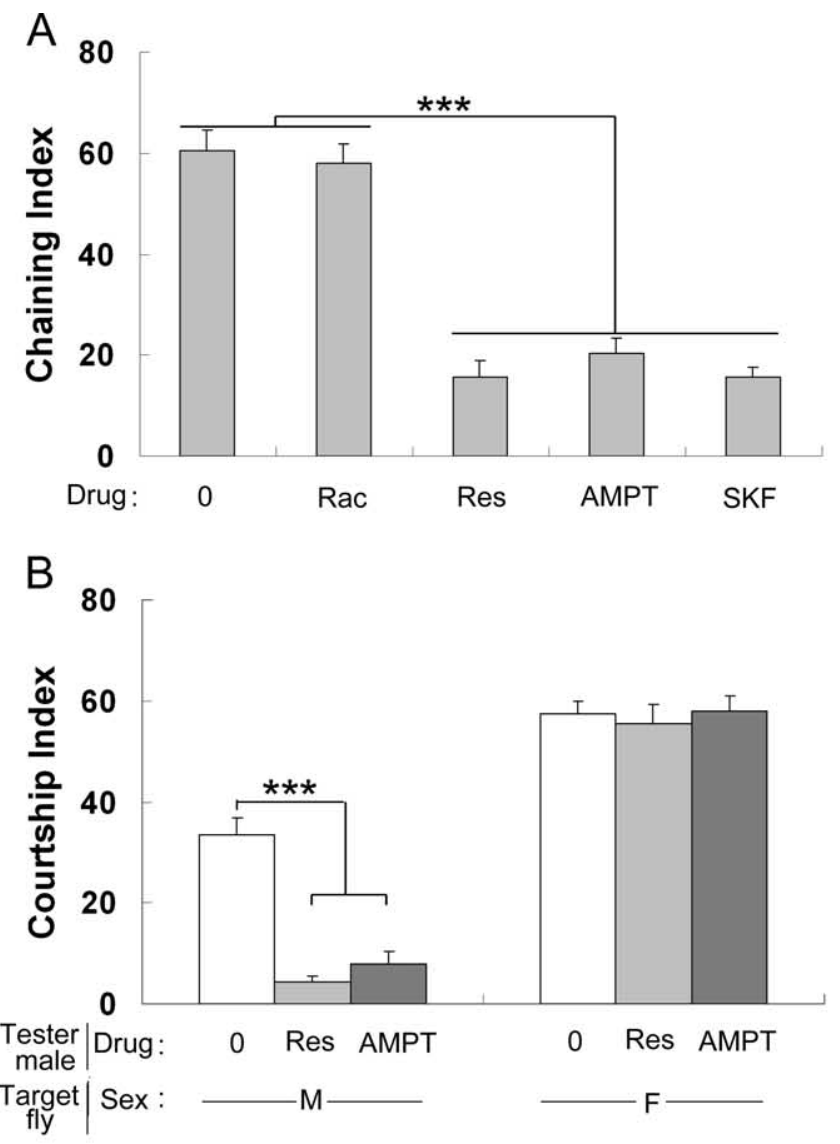

C

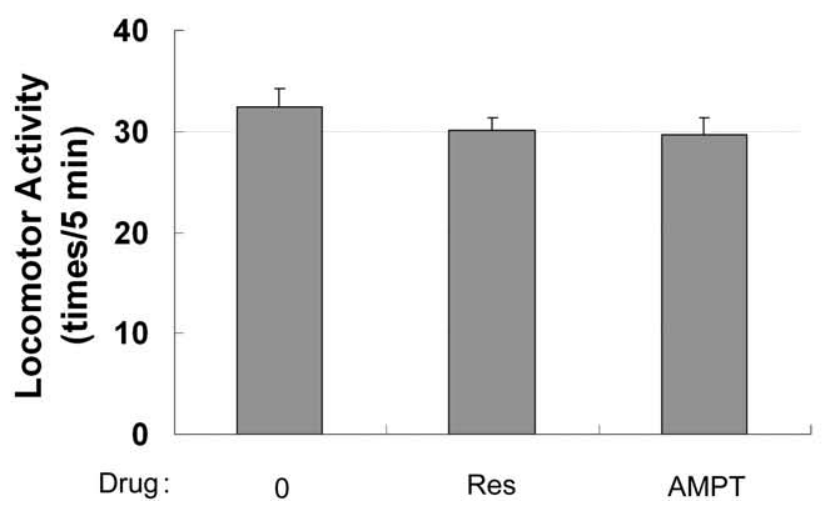

Figure 5. Effect of the increased DA level on male behavior. $\boldsymbol{A}-\mathbf{C}$ represent the $\mathrm{Chl}$, the $\mathrm{Cl}$, and the spontaneous locomotor activity of $T H-G A L 4$ NAS-TH males reared either on plain food (0) or in food supplemented with raclopride (Rac), reserpine (Res), AMPT, and SKF 83566 (SKF). Cl was measured toward single wild-type males (M) or virgin females (F). $n=28-43$, except for locomotor activity of Res-fed males $(n=21)$. Each histogram represents the mean behavioral activity with its SEM. ${ }^{* * *} p<0.001$ (for two-group comparison, Mann-Whitney rank sum test was used; for more than two-group comparison, ANOVA on rank test was used).

and raclopride, the antagonist of $\mathrm{D}_{2}$-like receptors (Yellman et al., 1997). Neither 10 nor $30 \mathrm{~mm}$ raclopride had effect on the ChI of TH-GAL4/UAS-TH males (Fig. $5 \mathrm{~A}$ for $10 \mathrm{~mm}$ ) (ChI of $66.20 \pm 2.2$ for $30 \mathrm{~mm}, n=39, p=0.21$, Mann-Whitney rank sum test). However, SKF 83566 significantly reduced the ChI of TH-GAL4/UAS-TH males (Fig. 5A). This indicates that $\mathrm{D}_{1}$ receptors but not $\mathrm{D}_{2}$ receptors may be involved in the variation of male-male courtship behavior induced by increased DA level. 


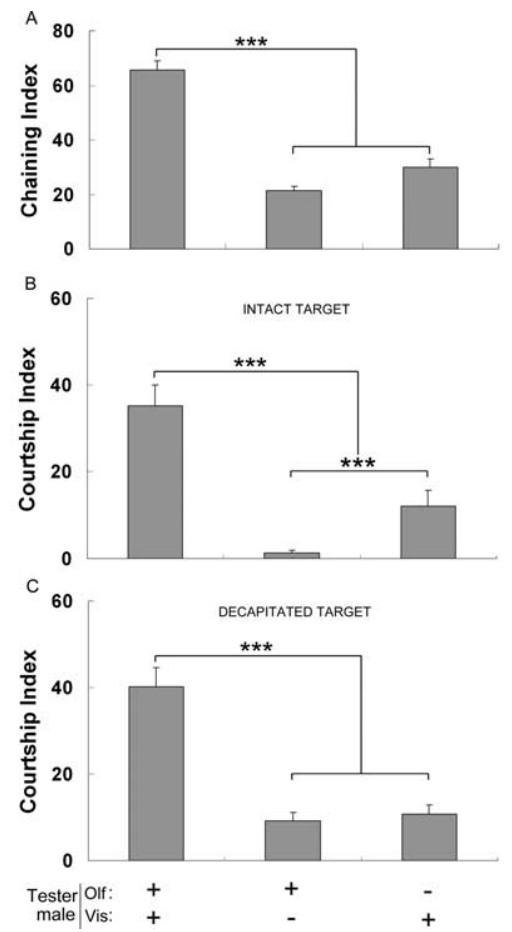

Figure 6. Consequence of sensory deprivation on the male-male courtship behavior of TH-GAL4/NAS-TH males. The manipulation of olfactory/auditory (Olf) and visual (Vis) stimuli is indicated at the bottom: + , presence; - , absence. $\boldsymbol{A}$, Chl for males with normal or altered sensory systems. $\boldsymbol{B}, \boldsymbol{C}, \boldsymbol{C l}$ for males with normal or altered sensory systems. Target males were either intact $(\boldsymbol{B})$ or decapitated $(\boldsymbol{C})$ wild-type males. Decapitation reduced the amount of acoustic stimuli produced by target males. $n=34-68$. Each histogram represents the mean behavioral activity with its SEM. ${ }^{* * *} p<0.001$ (Mann-Whitney rank sum test).

Alternatively, it is possible that both drugs do not target their respective receptors with a similar efficiency.

\section{DA-related male-male courtship behavior depends on sensory stimulation}

Drosophila male courtship and mate discrimination mostly depends on the perception of visual, pheromonal, and acoustic signals exchanged by flies (Yamamoto and Nakano, 1999; Greenspan and Ferveur, 2000). To assess their involvement in the increased intensity of male-male courtship behavior shown by TH-GAL4/UAS-TH males, we experimentally eliminated the effect of each type of signal and measured the behavioral effect (CI and ChI) following each type of sensory deprivation (Fig. 6). Tester males were either tested under red light in which they are virtually blind (Robertson, 1983), or ablated of both antennae and maxillary palps (NAM) to reduce their perception of volatile pheromones (Robertson, 1983) and of acoustic signals (Eberl et al., 1997). Decapitated target males were also used to reduce their emission of acoustic signals [decapitated males emit less or no acoustic signals (Paillette et al., 1991)].

Olfactory/auditory-deprived or visually deprived TH-GAL4/ UAS-TH males showed significantly reduced ChI (Fig. 6A) and CI to intact and to decapitated target males (Fig. 6B,C). The behavioral effect induced by either manipulation was so dramatic that it erased most of the effect induced on male-male CI. Although intact and decapitated males elicited very similar malemale CI to olfactory/auditory-deprived males, acoustic signals had a marginal but significant effect on visually deprived males: their CI with intact target was lower than that with decapitated target males ( $p<0.001$, Mann-Whitney rank sum test). Under white light, olfactory/auditory deprivation did not affect the courtship that $\mathrm{TH}-\mathrm{GAL} 4 / \mathrm{UAS}-\mathrm{TH}$ males directed to intact wildtype female targets (CI of $51.43 \pm 2.8$ for intact males, $n=34$; CI of $49.76 \pm 3.1$ for NAM males, $n=42$ ).

Therefore, the high male-male courtship shown by $\mathrm{TH}-$ GAL4/UAS-TH flies seems to mostly result from the abnormal perception of male inhibitory visual, olfactory, and acoustic signals that together normally tend to reduce male-male courtship behavior in wild-type males.

\section{Discussion \\ Increased amount of DA in DA cells enhances male propensity to court other males: developmental or functional effect?}

Increased DA amount in the brain of genetically and/or pharmacologically manipulated males was correlated with their increased propensity to court other wild-type males. This effect was clearly related to the targeting of the UAS-TH transgene (overexpression of tyrosine hydroxylase) in DA cells. Although $\mathrm{TH}-$ GAL4 driver is not active in all DA cells (Friggi-Grelin et al., 2003a, their Fig. 4), it was efficient enough to strongly increase both the amount of DA in the brain and the intensity of malemale courtship behavior of manipulated males. A recent paper also reported that dopamine is crucial for the ethanol-induced male-male courtship (Lee et al., 2008). Given that TH-GAL4 can drive $\mathrm{TH}$ overexpression in both nervous system and the hypoderm during all developmental stages (Friggi-Grelin et al., 2003a,b), we used elav-GAL4 to overexpress TH only in the nervous system: this was sufficient to strongly enhance male-male courtship. Because DA synthesized within the nervous system can be secreted out of nervous system to function elsewhere, the alteration of male-male courtship behavior may possibly result from the DA secreted out of the nervous system.

In TH-GAL4/UAS-TH males, as in $e^{l}$ mutant males, DA was kept at a high level during most developmental stages. These males showed much higher ChIs (Fig. $1 B, C$ ) than that of males fed with L-DOPA only during their adult life. This behavioral difference may be attributable to the effect of DA on the development of adult sensory organ. Similarly, our pharmacological attempt to decrease the level of DA and of male-male courtship only induced a partial effect: TH-GAL4/UAS-TH adult males fed with drugs only during adulthood showed a strongly decreased but not completely abolished male-male courtship behavior (Fig. $5 A, B)$. This indicates that increased DA level during preimaginal development also affects adult male-male courtship behavior. Therefore, the high amplitude of male-male courtship behavior shown by TH-GAL4/UAS-TH and $e^{I}$ males could result from the cumulative effects of DA elevation during both preimaginal and adult developmental stages. (1) The "preimaginal" effect could alter the development of adult sensory systems. This supports previous studies showing that that manipulation of DA during late larval developmental stage affects the formation of adult sensory nervous system (in particular, the visual system) (Neckameyer, 1996; Neckameyer et al., 2001). (2) The "adult" effect could alter the signaling role of DA in the male nervous system.

\section{Specific and sensory effects of DA on male behavior}

Our data indicate that increased DA synthesis during development had no general debilitating effect on behavior. Increased DA level in male brain was correlated with relatively specific behavioral defects: male-male courtship behavior was enhanced, whereas male-female courtship, general olfactory/gustatory response, and spontaneous locomotor activity remained unaltered. 
Moreover, although manipulated males showed reduced ability of discrimination between the sexes, they still clearly preferred females when they had the chance to choose between the sexes. This indicates that increased levels of DA strongly alter male perception of other male's sensory signals but have a weaken effect on male perception of female's sensory signals, which results in a courtship toward both females and males without drastic loss of sexual discrimination. A similar behavioral phenotype was induced by manipulating the level of extracellular glutamate in the synapses of the brain regions involved in pheromonal perception (Grosjean et al., 2008). In this case, males showed a stimulation instead of an avoidance toward 7-tricosene, the principal male contact pheromone. Given that the agonist of DA receptors affect the threshold of sex arousal (Andretic et al., 2005), the apparently unaffected male-female CI shown by TH-GAL4/UAS-TH males may be a ceiling effect because this level may be at its maximum. However, this is not the case, because TH-GAL4/UAS-TH males tested under red light showed a much lower CI of control females than wild-type males (respectively, $25.22 \pm 2.9, n=42$ and $42.24 \pm 4.3, n=33 ; p=0.001$, Student's $t$ test $)$.

Our results are similar to those of Andretic et al. (2005), which showed that males fed with agonist of DA receptor, methamphetamine, had an altered processing of visual signals.

The effect of DA on Drosophila locomotor activity was shown by pale mutant (Pendleton et al., 2002) and by fly fed with methamphetamine (Andretic et al., 2005), whereas DA function in fumin mutant did not alter spontaneous short-term locomotor activity (Kume et al., 2005). Our results showed that males with increased DA level displayed a normal spontaneous short-term locomotor activity, which was somewhat in accordance with the study of Kume et al. The contradiction of locomotor activity between our result and that of Andretic et al. may be attributable to different experimental manipulation. Two other studies demonstrated that DA depletion can affect two aspects of courtship behavior: female sexual receptivity and male courtship conditioning toward immature males (Neckameyer, 1998a,b). The fact that DA depletion did not change male-female courtship (Neckameyer, 1998a) but affected male-male courtship (our study) indicates that this manipulation induces sex-specific effect on courtship behavior.

Courtship behavior results from the coordination of a series of motor activities evoked in response to multiple sensory cues exchanged during courtship. Dopamine concentration and receptor activation have important roles in many behavioral situations (Schultz, 2002; Andretic et al., 2005). Dopamine can modulate neurotransmitter action on target neurons and coordinate the output of neuronal ensembles to generate behavioral patterns of varying complexity (Nusbaum et al., 2001; LeBeau et al., 2005). Dopamine is an important neuromodulator for Drosophila, and DA neurons have enormous fields of innervation covering essentially most neuropil regions of the fly brain (Monastirioti, 1999). This supports our results that DA neurons play a crucial role in integrating information from multiple sensory modalities.

The comparison between visually deprived or olfactory/ auditory-deprived TH-GAL4/UAS-TH males with control sibling males suggests that the strongly enhanced male-male courtship results from the altered perception of these sensory signals. Although male visual and olfactory stimuli seem to induce a strong effect on TH-GAL4/UAS-TH male-male courtship, acoustic signals (not emitted by decapitated targets) also play a role, yet with less importance compared with the effect induced by the two former sensory modalities. We do not know whether the intense male-male courtship behavior shown by manipulated flies is stimulated by male visual, olfactory, and auditory signals that are normally aversive (Greenspan and Ferveur, 2000) or whether these males have defective perception of their inhibitory effect. If the latter hypothesis is true, it means that, in the absence of these sensory signals, other yet unknown male sensory stimuli may be able to stimulate male courtship. Because NAM-operated TH-GAL4/UAS-TH males showed significantly decreased malemale courtship behavior in chaining and paired courtship assays, the strong male-male courtship behavior shown by intact $\mathrm{TH}-$ GAL4/UAS-TH males may not simply be attributable to the poor perception of aversive signals but rather to the perception of male olfactory/auditory signals that they find stimulating as in the case of genderblind mutant males toward the aversive male pheromone (Grosjean et al., 2008).

Although our data clearly allow us to rule out the effect of the mini-white gene $(\mathrm{mw})$ on the high male-male courtship behavior observed in our study, it is worth drawing a parallel with the high level of intermale courtship shown by pairs of $m w$ males, which tended to be also drastically reduced in the absence of visual cues (Hing and Carlson, 1996).

Our study reveals the intriguing effect of DA on Drosophila male-male courtship behavior. At the moment, we do not know whether our results can be generalized to other species. We do not know how the perturbation of DA precisely affects male-male courtship in Drosophila. Additional dissection of Drosophila male-male courtship may help us understand the fine mechanisms underlying sensory communication regulating interindividual behavior.

\section{References}

Acebes A, Grosjean Y, Everaerts C, Ferveur JF (2004) Cholinergic control of synchronized seminal emissions in Drosophila. Curr Biol 14:704-710.

Andretic R, van Swinderen B, Geenspan RJ (2005) Dopaminergic modulation of arousal in Drosophila. Curr Biol 15:1165-1175.

Anholt RR, Lyman RF, Mackay TF (1996) Effects of single P-element insertions on olfactory behavior in Drosophila melanogaster. Genetics 143:293-301.

Balakireva M, Stocker RF, Gendre N, Ferveur JF (1998) Voila, a new Drosophila courtship variant that affects the nervous system: behavioral, neural, and genetic characterization. J Neurosci 18:4335-4343.

Billeter JC, Villella A, Allendorfer JB, Dornan AJ, Richardson M, Gailey DA, Goodwin SF (2006) Isoform-specific control of male neuronal differentiation and behavior in Drosophila by the fruitless gene. Curr Biol 16:1063-1076.

Bradbury JW, Vehrencamp SL (1998) Principles of animal communication. Sunderland, MA: Sinauer.

Brand AH, Perrimon N (1993) Targeted gene expression as a means of altering cell fates and generating dominant phenotypes. Development 118:401-415.

Eberl DF, Duyk GM, Perrimon N (1997) A genetic screen for mutations that disrupt an auditory response in Drosophila melanogaster. Proc Natl Acad Sci USA 94:14837-14842.

Ferveur JF, Störtkuhl K, Stocker RF, Greenspan RJ (1995) Genetic feminization of brain structures and changed sexual orientation in male Drosophila melanogaster. Science 267:902-905.

Finley KD, Taylor BJ, Milstein M, McKeown M (1997) dissatisfaction, a gene involved in sex-specific behavior and neural development of Drosophila melanogaster. Proc Natl Acad Sci USA 94:913-918.

Friggi-Grelin F, Iché M, Birman S (2003a) Tissue-specific developmental requirements of Drosophila tyrosine hydroxylase isoforms. Genesis 35:175-184.

Friggi-Grelin F, Coulom H, Meller M, Gomez D, Hirsh J, Birman S (2003b) Targeted gene expression in Drosophila dopaminergic cells using regulatory sequences from tyrosine hydroxylase. J Neurobiol 54:618-627.

Gaines P, Tompkins L, Woodard CT, Carlson JR (2000) quick-to-court, a Drosophila mutant with elevated level of sexual behavior, is defective in a predicted coiled-coil protein. Genetics 154:1627-1637. 
Greenspan RJ, Ferveur JF (2000) Courtship in Drosophila. Annu Rev Genet 34:205-232.

Grosjean Y, Grillet M, Augustin H, Ferveur JF, Featherstone DE (2008) A glial amino-acid transporter controls synapse strength and homosexual courtship in Drosophila. Nat Neurosci 11:54-61.

Hall JC (1994) The mating of a fly. Science 264:1702-1714.

Heimbeck G, Bugnon V, Gendre N, Keller A, Stocker RF (2001) A central neural circuit for experience-independent olfactory and courtship behaviour in Drosophila melanogaster. Proc Natl Acad Sci USA 98:15336-15341.

Heisenberg M (2003) Mushroom body memoir: from maps to models. Nat Rev Neurosci 4:266-275.

Hing AL, Carlson JR (1996) Male-male courtship behavior induced by ectopic expression of the Drosophila white gene: role of sensory function and age. J Neurobiol 30:454-464.

Hull EM, Muschamp JW, Sato S (2004) Dopamine and serotonin: influences on male sexual behavior. Physiol Behav 83:291-307.

Kitamoto T (2002) Conditional disruption of synaptic transmission induces male-male courtship behavior in Drosophila. Proc Natl Acad Sci USA 99:13232-13237.

Kume K, Kume S, Park SK, Hirsh J, Jackson FR (2005) Dopamine is a regulator of arousal in the fruit fly. J Neurosci 25:7377-7384.

Lacaille F, Hiroi M, Twele R, Umemoto D, Inoshita T, Manière G, MarionPoll F, Osaki M, Francke W, Cobb M, Everaerts C, Tanimura T, Ferveur JF (2007) An inhibitory sex pheromone tastes bitter for Drosophila males. PloS One 15:e661.

Lasbleiz C, Ferveur JF, Everaerts C (2006) Courtship behavior of Drosophila melanogaster revisited. Anim Behav 72:1001-1012.

LeBeau FE, El Manira A, Griller S (2005) Tuning the network: modulation of neuronal microcircuits in the spinal cord and hippocampus. Trends Neurosci 28:552-561.

Lee HG, Kim YC, Dunning JS, Han KA (2008) Recurring ethanol exposure induces disinhibited courtship in Drosophila. PloS One 3:e1391.

Melis MR, Argiolas A (1995) Dopamine and sexual behavior. Neurosci Biobehav Rev 19:19-38.

McGuire SE, Le PT, Osborn AJ, Matsumoto K, Davis RL (2003) Spatiotemporal rescue of memory dysfunction in Drosophila. Science 302:1765-1768.

Monastirioti M (1999) Biogenic amine systems in the fruit fly Drosophila melanogaster. Microsc Res Tech 45:106-121.

Neckameyer W (1996) Multiple roles for dopamine in Drosophila development. Dev Biol 176:209-219.

Neckameyer W (1998a) Dopamine modulates female sexual receptivity in Drosophila melanogaster. J Neurogenet 12:101-114.

Neckameyer W (1998b) Dopamine and mushroom bodies in Drosophila: experience-dependent and -independent aspects of sexual behavior. Learn Mem 5:157-165.

Neckameyer W, O’Donnell J, Huang Z, Stark W (2001) Dopamine and sensory tissue development in Drosophila melanogaster. J Neurobiol 47:280-294.

Nusbaum MP, Blitz DM, Swensen AM, Wood D, Marde E (2001) The roles of co-transmission in neural network modulation. Trends Neurosci 24:146-154.

O’Dell KM, Armstong JD, Yang MY, Kaiser K (1995) Functional dissection of the Drosophila mushroom bodies by selective feminization of genetically defined subcompartments. Neuron 15:55-61.

Paillette M, Ikeda H, Jallon JM (1991) A new acoustic signal of the fruit-flies Drosophila simulans and D. melanogaster. Bioacoustics 3:247-254.

Pendleton RG, Rasheed A, Sardina T, Tully T, Hillman R (2002) Effects of tyrosine hydroxylase mutants on locomotor activity in Drosophila: a study in functional genomics. Behav Genet 32:89-94.

Pendleton RG, Rasheed A, Paluru P, Joyner J, Jerome N, Meyers RD, Hillman R (2005) A developmental role for catecholamines in Drosophila behavior. Pharmacol Biochem Behav 81:849-853.

Ramadan H, Alawi AA, Alawi MA (1993) Catecholamines in Drosophila melanogaster (wild type and ebony mutant) decuticalarized retinas and brains. Cell Biol Int 17:765-771.

Robertson HM (1983) Mating behavior and the evolution of Drosophila mauritiana. Evolution 37:1283-1293.

Robinow S, White L (1988) The locus elav of Drosophila melanogaster is expressed in all neurons at all developmental stages. Dev Biol 126:294-303.

Salvaterra PM, Kitamoto T (2001) Drosophila cholinergic neurons and processes visualized with Gal4/UAS-GFP. Brain Res Gene Expr Patterns 1:73-82.

Schultz W (2002) Getting formal with dopamine and reward. Neuron 36:241-263.

True JR, Edwards KA, Yamamoto D, Carroll SB (1999) Drosophila wing melanin patterns form by vein-dependent elaboration of enzymatic prepatterns. Curr Biol 9:1382-1391.

Villella A, Gailey DA, Berwald B, Ohshima S, Barnes PT, Hall JC (1997) Extended reproductive roles of the fruitless gene in Drosophila melanogaster revealed by behavioral analysis of new fru mutants. Genetics 147:1107-1130.

Wyatt TD (2003) Pheromones and animal behavior: communication by smell and taste. Cambridge, UK: Cambridge UP.

Yamamoto D, Nakano Y (1999) Sexual behavior mutants revisited: molecular and cellular basis of Drosophila mating. Cell Mol Life Sci 56:634-646.

Yamamoto D, Ito H, Fujitani K (1996) Genetic dissection of sexual orientation: behavioral, cellular, and molecular approaches in Drosophila melanogaster. Neurosci Res 26:95-107.

Yamamoto D, Jallon JM, Komatsu A (1997) Genetic dissection of sexual behavior in Drosophila melanogaster. Annu Rev Ent 42:551-585.

Ye YZ, Xi W, Peng YQ, Wang YZ, Guo AK (2005) Long-term but not shortterm blockade of dopamine release in Drosophila impairs orientation during flight in a visual attention paradigm. Eur J Neurosci 20:1001-1007.

Yellman C, Tao H, He B, Hirsh J (1997) Conserved and sexually dimorphic behavioral responses to biogenic amines in decapitated Drosophila. Proc Natl Acad Sci USA 94:4131-4136.

Zhang S, Odenwald WF (1995) Misexpression of the white ( $w$ ) gene triggers male-male courtship in Drosophila. Proc Natl Acad Sci USA 92:55255529. 